\title{
TENTH DISCUSSION SESSION
}

(Tuesday morning; 12 September, 1972)

\section{Chairman: D. M. POPPER}

Popper: As you know, there is going to be a brief summary of the highlights of our proceedings by Dr. Sahade. There is still time for anyone who wishes to make comments to do so, but I thought we'd get some kind of organization in the proceedings if we kept at first to a list of speakers. So I'll call on Dr. Lloyd Evans for his remarks.

Lloyd Evans: Plavec used evolutionary tracks by Iben to estimate the critical orbital period for which a star fills its Roche lobe at various evolutionary stages. The values for a star of $5 M_{\odot}$ are $10 \mathrm{~d}$ and $100 \mathrm{~d}$ at the Cepheid stage and the red giant tip, respectively. It is generally accepted that most Cepheids are in a post-red giant stage of evolution. The shortest known orbital period, $506 \mathrm{~d}$ for S Mus $(P=9 \mathrm{~d} .7)$, was found in an extensive survey of Cepheid radial velocities carried out at the Radcliffe Observatory. Given the considerable theoretical uncertainties, especially in red giant radii, and the small size of the sample ( 5 known periods, though another $\sim 5$ binaries almost certainly all have longer periods), agreement with the theoretical minimum $P \sim 100 \mathrm{~d}$ is satisfactory.

Plavec: The period of $100 \mathrm{~d}$ for a star of $5 M_{\odot}$ at the red-giant tip is the maximum period for mass exchange to take place before the star gets there. Any period above $100 \mathrm{~d}$ makes the system sufficiently wide for the stars to evolve independently. This is what you need to obtain a Cepheid.

Andersen: What is the amplitude of the orbital motion relative to that of the pulsations?

Lloyd Evans: They are roughly the same.

Batten: What is the latest estimate for binary frequency amongst Cepheids?

Lloyd Evans: Still about $15 \%$.

Hummer: Although the $\mathrm{N}$ III lines $\lambda \lambda 4634,40,41$ are seen in emission in the spectra of many types of objects including Of stars, for which they are classification lines, the mechanism for their formation has remained obscure. Because Otto Struve himself was one of the first people to discuss the problem of these lines (Swings and Struve, 1940) and because they are of some importance for the extended atmospheres of certain of the objects being discussed at this Symposium (for example, Cyg X-1)*, I would like to say a few words about some recent work explaining how these lines are formed. A full account of this work will appear shortly (Hummer and Mihalas, 1973). The multiplet in question is $3 d^{2} D-3 p^{2} P^{0}$. The subsequent transition $3 p^{2} P^{0}-3 s^{2} S$ is always seen in absorption, at least in O-type stars. The Bowen mechanism has sometimes been invoked as an explanation, but fails on several counts. Swings (1948) proposed that $3 d$ is pumped from the ground state $2 s^{2} 2 p^{2} P^{0}$ by continuum radiation

* See p. 110.

Batten (ed.), Extended Atmospheres and Circumstellar Matter in Spectroscopic Binary Systems, 277-285. All Rights Reserved. Copyright (C) 1973 by the IAU. 
which for Of stars peaks near the energy of the $2 p-3 d$ transition; $3 p$ is then drained by some unspecified mechanism. By solving the statistical equilibrium and radiative transfer equations for a model $\mathrm{N}$ III ion using Mihalas' recent non-LTE plane-parallel model atmospheres (NCAR Technical Note, NCAR-TN/STR-76; Boulder, Colorado), we showed that $3 d$ was populated primarily by dielectronic recombination and that $3 p$ was drained significantly by two-electron transitions to $2 s 2 p^{2}$ in addition to the other dipole-allowed transitions. Not only did the $\lambda 4640$ multiplet appear in emission for the values of effective temperature and surface gravity for which the emission is actually observed, but the equivalent widths were in accord with those measured by Peter Conti for Of stars in which $\lambda 4686$ was in absorption or neutralized, i.e. those designated as $\mathrm{O}(\mathrm{f})$ ) or $\mathrm{O}(\mathrm{f})$ by Walborn (1971). This comparison has already been published by Mihalas et al. (1972).

On the basis of our work, I would like to make three points. The first of these concerns the atmospheric structure of Of stars. We find that the $\mathrm{N}$ III lines, which are weak and therefore formed deep within the atmosphere, can be understood on the basis of a static, plane-parallel model, at least for those Of stars with $\lambda 4686$ in absorption. Yet for one of these objects, $\xi$ Per, Morton, Jenkins and Macy, (1972) observe strong ultra-violet lines that show the outer part of the atmosphere to be rapidly expanding. Our current picture is that as $\lambda 4686$ goes from absorption into emission and $\lambda 4640$ strengthens, (the progression $\mathrm{O}(\mathrm{f})) \rightarrow$ Of), the fraction of the atmosphere that is expanding rapidly increases, that is, the sonic point lies at increasingly greater depths in the atmosphere. Although in our calculations the mechanism proposed by Swings was not effective because the $2 p-3 d$ transition is saturated, we expect that as more of the atmosphere expands rapidly this transition will become desaturated and pumping by the continuum will add to the strength of the $\lambda 4640$ emission. Further work along these lines is in progress in Boulder by Castor, Mihalas and myself.

The second point is that our detailed understanding of the $\mathrm{N}$ III ion makes the lines of this spectrum available for use as temperature and density indicators for a wide variety of atmospheric conditions. More atomic parameters are now known for this ion than for any other (although our group at JILA is now producing atomic data to the same degree of completeness for other ions of $\mathrm{C}, \mathrm{N}, \mathrm{O}$ and $\mathrm{Si}$ ). Although a substantial amount of work is required to interpret the $\mathrm{N}$ III spectrum, our program could be used for any atmospheric model.

Finally, I would like to stress the danger of making inferences about the structure of an atmosphere from the appearance of emission lines without understanding the underlying physical mechanisms. The $\lambda 4640$ multiplet is seen in emission in Of stars only because of certain atomic properties of that ion, i.e. it is an intrinsic emission line, and does not imply mechanical heating, outward temperature rises, extended atmospheres or any of the other atmospheric features that are commonly associated with the appearance of emission lines.

Bolton: I agree with what you say about making errors if incorrect physics is used in the interpretation. In the case of Cyg X-1 I think that you must invoke something different from the particular non-LTE phenomena you have described here, even 
though you find some of the same lines found in the spectra of the Of stars. The primary star in Cyg X-1 lies outside the region of the $\mathrm{H}-\mathrm{R}$ diagram where the Of phenomena are found. The Cyg X-1 emission lines may arise from a similar process to that in the Of stars, but something else must be happening as well.

Hummer: I'm pretty sure that our proposed mechanism holds for all of these situations, although perhaps in the supergiants the Swings mechanism plays a more important role. I can see no way for the Bowen mechanism to work under these conditions.

Hutchings: Can you say whether these emissions will be enhanced in extended atmospheres?

Hummer: As the atmosphere becomes more and more extended, the streaming velocity of the gas increases and the $2 p-3 d$ transition is desaturated, so that the Swings mechanism almost certainly becomes effective. Of course the population of $3 d$ via dielectronic recombination continues to be effective until the temperature becomes too low.

Popper: But presumably both lines would be in emission in that case?

Hummer: We know of no cases of $\lambda 4097$ appearing in emission in the spectra of Of stars. In some of the objects in whose spectra it does appear, we know that the Bowen mechanism is operative.

Oliver: We have several times referred to the RS CVn class of binaries during this Symposium. I thought it might be useful to list the characteristics of the class. They are:

(i) Eclipsing binaries containing late-type undersized subgiant components.

(ii) They show $\mathrm{H}$ and $\mathrm{K}$ emission in the spectra of the secondary, or both, components. Six of these stars were first singled out by this characteristic (Struve, 1946). A larger list was published by Hiltner (1947).

(iii) They show two spectra. The mass ratios $q\left(m_{\text {cool }} / m_{\text {hot }}\right)$ range from about 0.8-1.3. Eggen (1955) called attention to the group and Popper has repeatedly mentioned them in recent years (e.g. Popper, 1970).

(iv) The secondary component is usually of spectral type $\mathrm{K} 0-\mathrm{K} 1 \mathrm{IV}$, the primary may range from F0-G5 IV or V.

(v) The light curves show both short-term and long-term variations.

(vi) There are fairly large and frequent period changes $\triangle P / P$ up to $10^{-3}$ or $10^{-4}$.

(vii) The secondary stars do not fill their Roche lobes (Plavec, 1967).

(viii) They generally show a wave-like distortion of the light curve. At some epochs this may make the maxima appear asymmetric, but the wave moves slowly toward earlier phases so as eventually to reverse the asymmetry. This was first seen in RS CVn itself as a result of long series of careful photometric observations at Catania (Catalano and Rodonò, 1967), but a similar feature is observed in the light curves of most, if not all, of the members of the class.

In Table I, I list some probable members of the class. Dr. Popper has kindly provided provisional values for the masses. The components are distinguished by the subscripts $h$ and $c$ - for hot and cool. 
Smak: I believe that these subgiants are not over-luminous.

Oliver: They are certainly not. It's hard to talk about the ratio of the luminosities without a good model for the $0^{\mathrm{m}} .25$ (wave-like) variation. If the secondary component is the source of the variation, it must be varying by considerably more than 0.25 - perhaps as much as 0.75 .

Popper: I think the fact that these are double-line binaries shows that the luminosities of the components are not very different; the masses are not very different - so obviously they are not very over-luminous.

TABLE I

Some Probable RS CVn Binaries

\begin{tabular}{llll}
\hline System & $\begin{array}{l}\text { Period } \\
\text { (d) }\end{array}$ & $\begin{array}{l}m_{h}+m_{c} \\
\left(M_{\odot}\right)\end{array}$ & $q=m$ \\
\hline SS Boo & 7.6 & 1.75 & 0.92 \\
RS CVn & 4.8 & 2.75 & 1.04 \\
WW Dra & 4.6 & 2.6 & 1.00 \\
Z Her & 3.99 & 2.3 & 0.90 \\
AR Lac & 1.98 & 2.6 & 0.99 \\
SZ Psc & 3.97 & 3.0 & 1.24 \\
RW UMa & 7.33 & 2.2 & 1.00
\end{tabular}

Hall: I want to talk about the evolutionary status of these RS CVn binaries and about some of the problems which arise in understanding their physical nature. I have already said (p. 143) that I think these binaries are in some sort of pre-main-sequence evolution (Hall, 1972). Although this is difficult to prove conclusively (the evidence must be regarded as somewhat circumstantial) it is much more tenable than either of the two other possible interpretations, namely post-main-sequence evolution before or after mass transfer. Serious problems remain, however, even if my basic interpretation is correct.

A pre-main-sequence interpretation can best explain why both stars are above the main-sequence but are both detached; why stars around $1 M_{\odot}$ (in some, like SS Boo, the two stars are even less massive) can appear 'evolved', when the galaxy is not old enough for them to have evolved off the main sequence; and why one star is losing mass at a very rapid rate even though it is definitely not filling its Roche lobe. This interpretation can best explain the very strong $\mathrm{H}$ and $\mathrm{K}$ emission, which we associate with youthful chromospheric activity; the contact components in bona fide Algol-like systems are also subgiants, quite similar in spectral type and radius, but do not display this anomalously strong $\mathrm{H}$ and $\mathrm{K}$ emissions. And it has the least difficulty in explaining the fact that the more 'evolved' component can be either the more or the less massive one.

There are two additional pieces of evidence, not yet published, which support the pre-main-sequence interpretation. First, $J H K L$ photometry of six of these systems shows that there is an infra-red excess of about 0.5 , probably in the cooler component. 
Furthermore, $U B V$ photometry suggests the possibility of a slight ultra-violet excess in some of the systems. Then we have an energy distribution which cannot be fitted by any one spectral type. A similar situation exists with the T Tau stars, although the infra-red excess in the T Tau stars is larger, about $2^{\mathrm{m}}$ or more at $L$. Thus the energy distribution is consistent with my claim that the systems are young, but not so extremely young as the T Tau stars themselves. The effective temperature derived from consideration of the entire spectrum from $U$ to $L$ is around $1000 \mathrm{~K}$ cooler than that derived from the $B-V$ colour index alone, or the spectral type, which invariably is quite close to K0. A K0 star would be too hot for the cooler component to be on the Hayashi track for its mass, but an effective temperature about $1000 \mathrm{~K}$ cooler is acceptable. I have also mentioned before (p. 143) Montle's result that the average distance of these binaries from the galactic plane is about 80 parsecs, suggestive of an age of about 6 to $10 \times 10^{7}$ yrs. The average distance from the plane of Algol-like systems with a total mass less than $2.75 M_{\odot}$ is about 155 parsecs, suggestive of an age of about 3 to $7 \times 10^{8} \mathrm{yrs}$. The dispersion of velocities perpendicular to the galactic plane $\left\langle Z^{2}\right\rangle^{1 / 2}$, is about $12.5 \mathrm{~km} \mathrm{~s}^{-1} \pm 2 \mathrm{~km} \mathrm{~s}^{-1}$ for the RS CVn systems, suggestive of an age of about 1 to $3 \times 10^{8} \mathrm{yrs}$, and $15.5 \mathrm{~km} \mathrm{~s}^{-1} \pm 4 \mathrm{~km} \mathrm{~s}^{-1}$ for the Algol-like systems.

There seems to me to be many reasons why we should blame the wave-like distortions of the light-curve on an uneven distribution of surface brightness on the cooler star. One side of the cooler star seems to be fainter than the other. Why this should be so is unclear. The fainter side of the cooler star slowly changes its orientation with respect to the other star, executing one complete migration approximately every $10 \mathrm{yr}$. There is a good correlation between the orientation of the fainter side and the large period increases and decreases observed in the $(\mathrm{O}-\mathrm{C})$ diagram. This correlation is best explained as a result of mass loss, actually angular-momentum loss from the fainter side, since the binary speeds up when the fainter side is on the trailing hemisphere, and slows down when the fainter side is on the leading hemisphere. Eugene Milone and I have found the same correlation in RT Lac, another RS CVn-type system. In my paper I supposed that the fainter side was darkened by sunspots and that flare activity accompanying these spots might account for the outflow of matter. However, another interpretation should be explored. The energy equivalent of the required rate of angular-momentum loss is quite large compared to the luminosity of the cooler star. Perhaps we should say that the fainter side is fainter because it is losing mass, and only ask why it is losing mass. Using very simple assumptions, I found that $10^{-6} M_{\odot}$ of mass lost each year at an ejection velocity of $3000 \mathrm{~km} \mathrm{~s}^{-1}$ could account for the observed period changes. If there is a strong magnetic field, the mass could be lost from the ends of long magnetic 'spokes'. A lower rate of mass loss and/or velocity of ejection would then give the same rate of loss of angular momentum.

Oliver: My list was not meant to be complete - just a sample of seven probable members of this class. Essentially all double-line late-type systems showing $\mathrm{H}$ and $\mathrm{K}$ emission in their spectra could be included. Virtually all systems of that type listed by Popper seem to belong to the class - we know of at least two dozen members right now. Perhaps there could be some argument about RT Lac. 
Smak: I believe that the pre-main-sequence explanation is a very promising one, even if you do have to drop out those systems for which the mass ratios are inconsistent...

Hall: No, you don't have to. The mass loss itself gets over that problem - if it's at anything like the rate I claim. If the mass loss goes on for any length of time, the K0 IV star will soon become the less massive - certainly by the time it reaches the main sequence. That's how I get around the problem of the (presently) more massive secondary.

Smak: Then you might find that the primary went through the same phase that the secondary is now in, some time before.

Hall: That's the best way to think of the situation at present, but perhaps the primary and secondary evolve differently right from the beginning.

Smak: If we consider the contraction phase, it is quite possible that the secret lies more deeply, namely in the early history of the system.

Hall: I think these stars are suffering the effects of an extremely traumatic birth. We should not think of a simple 'interaction effect': the systems are suffering from their past history.

Plavec: Dr. Hall seems to believe that if $\mathrm{H}$ and $\mathrm{K}$ emission is observed in Algoltype systems it is inevitably associated with the disk around the primary component. This is only partially true. It's true that when the emission lines of the ring are extremely strong you observe also double or single emission in $\mathrm{H}$ and $\mathrm{K}$. But, for example, the spectrum of $U$ Sge discloses a reversal inside the deep absorption of $\mathrm{K}$ lines of the secondary spectrum. But the subgiants themselves do show emission of the same type as these R CVn systems. The difference only might be in the intensity of the line, and $\mathrm{I}$ would say that in those systems $\mathrm{H}$ and $\mathrm{K}$ emissions are particularly strong. But basically, they are no different from subgiants in Algol systems.

Hall: I have to reply to this. The Wilson-Bappu effect, and the apparent universality of its application, would tell you that any subgiant between late $G$ and early $K$ in spectral type must show some $\mathrm{H}$ and $\mathrm{K}$ emission. It would seem to me that the $\mathrm{H}$ and $\mathrm{K}$ emission arising from the subgiant component itself in Algol-like systems is probably just what would be expected from the Wilson-Bappu effect. The emission from the K0 IV component in the RS CVn systems, in contrast, is very much stronger. I can thank Dr. Popper for making this clear to me (although I don't want to hold him responsible for the things I say). About a year ago I was trying to interpret the RS CVn systems as post-main-sequence objects, i.e., similar to the Algol-like systems. Within that framework I could not easily understand the $\mathrm{H}$ and $\mathrm{K}$ emission and therefore wanted to dismiss it as simply Wilson-Bappu emission. But Dr. Popper emphasized to me how very much stronger the emission was in the RS CVn systems.

Batten: In the spectrum of the secondary component of $U$ Cep, even though the emission is not strong enough to come above the continuum, it is definitely much stronger than you would expect from the Wilson-Bappu effect.

Popper: When you talked of an average value of $z$, did you make any allowance 
ior the fact that those two groups of stars have systematically different luminosities?

Hall: Incompleteness can certainly have an important effect on the mean value derived for $z$. We have done two things. First we plotted the value of $z$ versus $d \cos b$, thereby producing a side view or cross-section of the galaxy, so to speak. Examination of the mean $z$ within various vertical sections assured us that it remained more or less constant as a function of $z \cos b$. Since the sample becomes more incomplete with increasing $z \cos b$ and since the mean value of $z$ was not obviously correlated with $z \cos b$, we concluded tentatively that our mean value for $z$ was not grossly in error. Second, we noticed that a concentration of interstellar absorption towards the galactic plane, along with the fact that obscuration contributes to incompleteness, would have the effect of exaggerating the derived mean value of $z$.

This doesn't completely answer your questions but, because the effect of incompleteness on the statistics is a crucial question, Montle is now working to remove the effect explicitly, in a more formal way. Until this is done, we do not want to publish any results.

D. B. Wood: As I listened to the first few days of this Symposium, the following idea struck me. We are at a stage of understanding the photometry of eclipsing stars that is comparable to that reached in the study of planetary motions in the 16th Century. We find ourselves with the necessity of inventing 'epicycles' to explain our observations - that is, we need gas streams, bumps, hot spots, and what have you. Instead of calling upon these various devices to explain deviations from the model of eclipsing stars, it seems particularly obvious that we should change the model. In this context, let me show you a specific example of what rectification does.

Rectification treats stars as ellipsoids with concentric isophotes. In reality, however, the isophotes on such a star will be distorted toward one end, except at phases $0^{\circ}, 90^{\circ}$, $180^{\circ}$ and $270^{\circ}$. Thus rectification will leave behind an apparent 'hot spot'. The effect of this spot will be to increase system brightness away from quadrature, until this brighter area is eclipsed. This phenomenon will look very much like what Dr. Walter has shown us. The amplitude of this effect would depend strongly upon the amount of distortion of the stars.

Walter: This is not quite clear. The hot spot is attributed to the primary component of Algol-type systems. The primary star is very nearly spherical, and there is no problem with the distribution of gravity darkening and limb darkening.

D. B. Wood: Well, another problem with rectification, especially applied to the Algol-type system, is that there's only one distortion parameter. If you have a very distorted cool star and a nearly spherical hot star, the distortion is averaged over the whole system. The effect of rectification is thus to treat the hot star as though it is more distorted than it really is.

Popper: I think the only way one could resolve this controversy would be for someone like Dr. Wood to use his techniques to analyze Dr. Walter's observations. Then Dr. Wood and Dr. Walter could compare their results and see whether, as a matter of fact, there were any outstanding differences. It appears that Dr. Wa'ter has allowed for first-order effects of the difference in shapes of the components. I think that with- 
out some sort of comparison of that kind, further discussion a: to whether Dr. Walter's technique is adequate will not be useful at this time.

Herczeg: Many of us participated in the non-recorded session dealing with $\beta$ Lyr the other day. Although we certainly did not solve any of the major problems concerning this famous system, we were able to point out 4 or 5 current problems, the solution of which may be of some importance for future investigations. I will try to give a summary of these problems:

(1) We had the impression that the $\mathrm{H} / \mathrm{He}$ ratio in the system is still a rather uncertain quantity although underabundance of hydrogen has been proposed by several authors. Perhaps we should try to re-examine this question using the best spectra and the best theory even if strong disturbances in the system make an exact solution almost impossible.

(2) It would be desirable to obtain a new photometric solution based on the methods of light-curve synthesis. The question of the secondary eclipse - how much light and of what colour is lost at phase 0.5 - is of particular interest.

(3) The primary eclipse should be observed and reobserved faithfully (in several colours), not only at a few points but if possible rather continuously covered throughout its whole width, since permanent or recurrent features may shed light on the nature of the eclipsing body.

(4) We have quite a few sets of polarimetric observations that should be assembled and rediscussed under the following aspect: do they confirm any of the published models? We find many quotations and remarks in the literature to this effect but a thorough and critical comparison is still wanting.

(5) There was consensus among participants that publication (or making accessible) of the OAO observations of $\beta$ Lyr in the near future would help our efforts considerably and is highly desirable.

Perhaps I am allowed to move that Drs. Batten and Sahade, organizers of the last year's co-ordinated program, should contact people responsible for the OAO observation and convey this question of ours to them.

Underhill: You forget, to obtain an abundance ratio you have to solve a serious problem in radiative transfer in a way that will be valid for a stream of helium.

Herczeg: We only want approximate data in order to decide, eventually, whether the B8 star is normal or peculiar.

Devinney: Perhaps some spectral scanning of $\beta$ Lyr would be useful.

Underhill: A lot of OAO material is now being placed in a data bank. When I go back to Goddard, I will make inquiries for you.

(After the close of the Symposium, Dr. Underhill sent a copy of the following memorandum from Dr. C. D. Wende of NASA, with the request that it be included in the Proceedings. The memorandum was dated 20 September, 1972.)

The following is the current status of OAO-2 data in the National Space Science Data Center:

$S A O$ Data: The final, revised version of the Smithsonian Celescope star catalog is expected to arrive today. This catalog will be available both on magnetic tape and on microfilm. The documen- 
tation appears to be complete, and these data should be available from the NSSDC for general requests by late October.

WEP Data: The NSSDC has received approximately 135 reduced data tapes out of an expected total of 400 (note: these are arriving at the expected rate). Other WEP data, expected later, include microfilm copies of the contents of the reduced data tapes, microfilmed plots of the spectrometer data, and magnetic tapes of the reduced spectrometer data. Note that the reduced data tapes contain reduced (i.e., instrumentally corrected) photometer data, but unreduced spectrometer data. Data on the reduced data tapes (and the corresponding microfilm) are in chronological order by the date of observation; observations of specific objects must first be located in a catalog of observations, and then these observations may be found in the data tapes/microfilms. User documentation is being written by Wisconsin, but the completion date is as yet unknown. This documentation is the pacing item as far as release of these data are concerned.

Scientists desiring copies of these data should write to:

\author{
National Space Science Data Center \\ code 601.4 \\ Goddard Space Flight Center \\ Greenbelt, Maryland, 20771.
}

Scientists who are not U.S. citizens also have access to these data by writing:

\author{
World Data Center A, for Rockets and Satellites \\ Code 601 \\ Goddard Space Flight Center \\ Greenbelt, Maryland, 20771, U.S.A.
}

Persons desiring these data need not wait until its availability is formally announced, but may write to one of the above addresses, their names will be filed, and they will be notified as soon as the data are released.

\title{
References
}

Catalano, S. and Rodonò, M.: 1967, Mem. Soc. Astr. Ital. 38, 395.

Eggen, O. J.: 1955, Publ. Astron. Soc. Pacific 67, 315.

Hiltner, W. A.: 1947, Astrophys. J. 106, 481.

Hummer, D. G. and Mihalas, D.: 1973, Astrophys. J. (in press).

Mihalas, D., Hummer, D. G., and Conti, P.: 1972, Astrophys. J. Letters 175, L99.

Morton, D. C., Jenkins, E. B., and Macy, W. W.: 1972, Astrophys. J. 177, 235.

Plavec, M.: 1967, Bull. Astron. Inst. Czech. 18, 334.

Popper, D. M.: 1970, in K. Gyldenkerne and R. M. West (eds.), Mass Loss and Evolution in Close Binaries, Copenhagen Univ. Press, p. 13.

Struve, O.: 1946, Ann. Astrophys. 9, 1.

Swings, P.: 1948, Ann. Astrophys. 11, 228.

Swings, P. and Struve, O.: 1940, Astrophys. J. $91,546$.

Walborn, N. R.: 1971, Astrophys. J. Suppl. 23, 257. 